\title{
Effects of the First COVID-19 Lockdown on Ophthalmological Patient Care
}

\section{Die Auswirkungen des ersten COVID-19-Lockdowns auf die Patientenversorgung im Bereich der Augenheilkunde}

Authors

Anna Schuh ${ }^{1}$, Stefan Kassumeh ${ }^{1}$, Valerie Schmelter ${ }^{1}$, Lilian Demberg ${ }^{1}$, Jakob Siedlecki ${ }^{\circledR}$, Andreas Anschütz ${ }^{1}$, Thomas Kreutzer ${ }^{1}$, Wolfgang J. Mayer ${ }^{1}{ }^{\mathbb{D}}$, Thomas Kohnen ${ }^{2}$, Mehdi Shajari ${ }^{1,2}$, Siegfried Priglinger $^{1}$

Affiliations

1 Augenklinik und Poliklinik, Klinikum der Universität München, LMU München, Germany

2 Augenklinik, Goethe-Universität Frankfurt am Main, Frankfurt am Main, Germany

Key words

Covid-19, Corona Virus, pandemic, lockdown

Schlüsselwörter

COVID-19, Coronavirus, Pandemie, Lockdown

$\begin{array}{ll}\text { received } & 23.2 .2021 \\ \text { accepted } & 14.6 .2021 \\ \text { published online } & 15.9 .2021\end{array}$

Bibliography

Klin Monatsbl Augenheilkd 2021; 238: 1220-1228

DOI 10.1055/a-1529-6726

ISSN 0023-2165

(C) 2021. Thieme. All rights reserved.

Georg Thieme Verlag KG, Rüdigerstraße 14,

70469 Stuttgart, Germany

Correspondence

PD Mehdi Shajari

Klinikum der Universität München, LMU München, Augenklinik und Poliklinik

Mathildenstraße 8, 80336 München, Germany

Phone: + 49(0) 89440053811 , Fax: + 49(0) 89440055160

gmsams2020@gmail.com

Supplementary material is available under

https://doi.org/10.1055/a-1529-6726

\section{ABSTRACT}

Purpose To determine the effect of lockdown on medical care, with the example of ophthalmology.

Methods Patients in a period during the first lockdown were compared to a non-lockdown period, with a total of 12259 patients included in an observational study. Changes in different areas (elective, emergency, inpatients, surgeries) and eye care subspecialties were compared. Emergency patients were analyzed according to severity and urgency. Patients showing hints requiring treatment for urgent cardiovascular diseases were determined. Differences in patients who would have suffered severe vision loss without treatment were identified and the QALY (quality-adjusted life years) loss was determined accordingly. A model to prioritize patient visits after the end of lockdown or in future lockdown scenarios was developed. Data were collected at the University Eye Hospital LMU Munich and patient files were reviewed individually by ophthalmologists.

Results The average patient number decreased by $-59.4 \%$ $(p<0.001)$, with a significant loss in all areas (elective, emergency, inpatients, surgeries; $p<0.001$ ). There was a decline of $-39.6 \%$ for patients at high risk/high severity. Patients with indications of a risk factor of future stroke declined significantly $(p=0.003)$. QALY loss at the university eye hospital was 171, which was estimated to be 3160-24143 for all of Germany. Working up high losses of outpatients during these 8 weeks of projected lockdown in Germany would take 7-23 weeks under normal circumstances, depending on ophthalmologist density. The prioritization model can reduce morbidity by up to $78 \%$.

Conclusion There was marked loss of emergency cases and patients with chronic diseases. Making up for the losses in examinations and treatments will theoretically take weeks to months. To reduce the risk of morbidity, we recommend a prioritization model for rescheduling and future lockdown scenarios.

\section{ZUSAMMENFASSUNG}

Hintergrund Erfassung der Auswirkungen eines Lockdowns auf die medizinische Versorgung am Beispiel der Augenheilkunde.

Methoden In einer Beobachtungsstudie wurden Patienten in einem Zeitraum während des ersten Lockdowns mit einem Zeitraum ohne Lockdown verglichen. Hierbei wurden insgesamt 12259 Patienten eingeschlossen. Es wurden Veränderungen in verschiedenen Bereichen (elektive Fälle, Notfälle, stationäre Fälle, Operationen) und augenärztlichen Subspezialitäten verglichen. Notfallpatienten wurden nach Schwere- 
grad und Dringlichkeit analysiert. Patienten mit ophthalmologischen Erkrankungen, die einen Hinweis auf behandlungsbedürftige, dringende kardiovaskuläre Erkrankungen darstellen, wurden ermittelt. Unterschiede von Patienten, die ohne Behandlung einen schweren Sehverlust erlitten hätten, wurden identifiziert und der QALY-Verlust entsprechend ermittelt. Ein Modell zur Priorisierung von Patientenbesuchen nach dem Ende des Lockdowns oder in zukünftigen Lockdownszenarien wurde entwickelt. Die Daten wurden an der Universitätsaugenklinik LMU München erhoben und die Patientenakten einzeln von Augenärzten geprüft.

Ergebnisse Die durchschnittliche Patientenzahl sank um - 59,4\% $(p<0,001)$ mit einem signifikanten Verlust in allen Bereichen (elektive Fälle, Notfälle, stationäre Fälle, Operationen; $\mathrm{p}<0,001)$. Bei Patienten mit hohem Risiko/hohem Schweregrad ergab sich ein Rückgang von - 39,6\%. Patienten mit ophthalmologischen Erkrankungen, die einen Hinweis auf ein mögliches erhöhtes Risiko für einen zukünftigen Schlaganfall geben, nahmen signifikant ab $(p=0,003)$. Der QALYVerlust an der Universitätsaugenklinik betrug 171, geschätzt für Deutschland 3160-24143. Die Aufarbeitung der hohen Verluste an ambulanten Patienten während der 8 Wochen Sperrung würde auf Deutschland hochgerechnet unter normalen Umständen je nach Augenarztdichte 7-23 Wochen dauern. Das Priorisierungsmodell kann die Morbidität um bis zu $78 \%$ reduzieren.

Fazit Es kam zu eminenten Verlusten sowohl bei Notfallpatienten als auch bei Patienten mit chronischen Erkrankungen. Das Aufarbeiten der verpassten Untersuchungen und Behandlungen wird theoretisch Wochen bis Monate dauern. Um das Morbiditätsrisiko zu reduzieren, empfehlen wir ein Priorisierungsmodell für die Wiedereinbestellung nach dem Lockdown und künftige Lockdownszenarien.

\section{Introduction}

In times of international crisis, politicians are forced to make crucial decisions regarding social and individual human welfare. The COVID-19 pandemic started as an unprecedented crisis of our time and had, as all catastrophes, its own particular character. There was no historical blueprint in modern civilization that could be used to properly react to this pandemic. Not knowing what will be ahead, most governments decided to conduct a lockdown in order to restrict the spread of the virus. In Germany, the first lockdown period lasted 7 weeks (22.03. to 04.05 .2020$)$. Already in the beginning, the medical benefit of this step was often discussed against the economic harm it causes. What was and is still required, however, is a scientifically led discussion not just on the benefits of "flattening the curve" through mass quarantining, but also the harm the lockdown causes to our health by restricting medical care [1,2]. Especially in the first weeks of lockdown, many patients suffered from not being treated for their diseases due to the lockdown restrictions and because of this may have to live with irreversible health consequences. To better understand the impact of the lockdown on medical care, its effects were analyzed at the largest single-center eye hospital in Germany. Due to the high number of outpatients, as well as inpatients, and including many diseases, which require an interdisciplinary treatment approach in ophthalmology (e.g., retinal arterial occlusion - neurologist, uveitis - rheumatologist, diabetic retinopathy - endocrinologist, etc.), implications could be estimated for acute and chronic eye care as well as interdisciplinary patient care. Furthermore, a prioritization model is presented to decrease overall population morbidity caused by the backlog of untreated patients due to the mandatory shutdown.

\section{Methods}

Permission to conduct the study was obtained by the institutional review board of Ludwig-Maximilians-University Munich (Ethikkommission LMU München). The same review board declared, that due to the retrospective nature of the study, informed patient consent was not necessary. The Declaration of Helsinki and its ethical principles were followed. Data was collected at the University Eye Hospital Munich (60 ophthalmologists/54 full-time equivalents, corresponding to $5.18 \%$ of 1042 in-hospital ophthalmologists in Germany). In calculations evaluating the pan-German situation, measured quantities were projected on the total number of ophthalmologists (7639 - according to the German Medical Association) [3] or only on the number of in-hospital ophthalmologists depending on the specific question.

The eye hospital is located in the center of Munich and $10 \mathrm{~km}$ away from the main hospital buildings where COVID-19 patients were treated during the analyzed period. With the start of the internal hospital lockdown on March 14, 2020 (1 week before the official lockdown of the federal state of Bavaria), most elective appointments (except follow up of critical cases e.g. post-operative follow-ups, follow-ups of emergency patients and tumor patients, ongoing IVT [intravitreal] treatment) and surgeries were cancelled and a triage station where patients were checked for possible COVID-19 infection signs was installed the hospital entrance. Furthermore, measures were taken to reduce the viral spread of the infection (increasing hygiene measures, phone consultation, no visits, etc.).

\section{Patient decline}

To evaluate reasons for the patient decline, we compared ophthalmology patient numbers to number of infections in Germany [4]. Furthermore, as patients may change their behavior due to a rise of infections in other regions outside of Germany, we compared patient numbers to the number of searches of the term "corona virus" on Google via Google Trends [5]. The analyzed period of time stretched from 20.01.2020 to 26.04.2020. For deeper analysis and better comparison to the regular setting, we included all patients seen between 16.03.2020 and 12.04.2020 (COVID Period, CP) to all patients seen between 25.03.2019 and 21.04.2019 (non-COVID Period, NCP). The NCP was postponed by 8 days in order to have an equal number of working days, weekends, and 
public holidays in both groups. Patients over 60 years were classified as COVID-19 high-risk patients. To avoid systematic errors by automatic analysis of patient visits, all patient files during NCP and CP were reviewed by one of five ophthalmologists (M.S., A.S., S.K., V.S., L.D.). Subgroup analysis was performed based on ophthalmic subspecialties (e.g., glaucoma) and medical care units (e.g., elective care).

\section{Assessment of patient care}

Two senior ophthalmology consultants (A.S. and M.S.) categorized each emergency patient during $\mathrm{CP}$ and NCP based on the patients' charts individually and compared their results (83\% initial agreement level). In cases of discrepancy, an agreement was reached after discussion. The categorization was based on potential severity of visual decline (low: $\leq 1$ line on Snellen chart, medium: 2 lines, high: $\geq 3$ lines) or on meaningful impact on general health (e.g., stroke) and the likelihood of deterioration (1: low probability, 2: medium probability, 3: high probability) in case of delay of the ophthalmological examination or treatment for 1 week. Information on visual acuity is in decimals.

Emergency patients in whom the weak eye was at least moderately impaired (according to WHO [6] visual acuity of 0.3 ) and the better eye had a pathology that would lead to a visual acuity worse than that of the weak eye if left untreated (meaning the patient would be disabled due to binocular moderate visual impairment $\leqslant 0.3$, severe impairment $\leqslant 0.1$, or blindness $\leqslant 0.05$ ) within a week were identified by A.S. and M.S. (initial agreement level $71 \%$ ). Quality-adjusted life year (QALY = year of life*utility value) was calculated by the difference in the age of the patient to average life expectancy in Germany (80.8 years). To further calculate the productivity loss (according to Wittenborn [7] \$50000/QA$L Y \cong € 45670 / Q A L Y$ ), the difference to retirement age (67 years) was calculated. The utility value [8] was calculated with $U=$ $(0.374)$ (visual acuity in better seeing eye) +0.514 . Furthermore, diseases forming a vascular ophthalmologic event were counted and classified as mild (hyposphagma), moderate [central retinal vein occlusion, branch retinal vein occlusion, branch retinal artery occlusion, non-arteriitic anterior ischemic optic neuropathy (NAION)], and severe (central retinal artery occlusion, transient ischemic attack (TIA) hints for general events (e.g., stroke).

To assess chronic ophthalmologic diseases, the number of patient visits and executed operations for glaucoma, diabetic retinopathy (DR), and age-related macular degeneration (AMD) were analyzed. The number of weeks required to see all lost patients (NCP-CP) under normal circumstances was analyzed by federal states in Germany (supplement A).

\section{Simulation model}

Development of a simulation model in order to decrease overall morbidity through prioritization of patient visits during a rescheduling period or a possible future lockdown scenario was established (detailed description in supplement B) for a single incident (like, e.g., stroke) and chronic diseases (like, e.g., diabetes) with two scenarios:

(1) scenario $1_{\text {score }}=$ severity

(2) scenario $2_{\text {score }}=$ severity $\times$ urgency
To simulate the different progression types, we used the following functional formula, which reflects the progression of the severity of the illness, as a function of time $t$, and the speed of progression of the illness $\alpha$, as well as the maximal severity of the illness:

(3) severity $=\left(1-e^{-\alpha t}\right) \times$ severity $_{\max }$

In our simulation study, $\alpha \in\{0.1 ; 0.2 ; 0.4\}$ reflecting a slow, medium, and fast progress of the illness, and $t \in[0 ; 30]$ reflecting 30 time periods. We differentiate between low, medium, and high maximal severity of the illness, severity $\max \in\{1 ; 2 ; 3\}$.

As it was a simultation, criteria for the different progress rates and severity levels were not defined. Euler's number was chosen as the constant to estimate the progression of a pathology, as it is found in many natural processes. The calculation of the correct constant was not feasible as this would have required a different patient cohort for each pathology and as this was not the primary interest of this study a general assumption was accepted in this matter.

\section{Statistical analysis}

Patients' characteristics and outcomes were compared with the use of independent t-tests or Mann-Whitney $U$ test for continuous variables and chi-square tests or Fisher's exact test for categorical variables. Poisson regression analysis was used to calculate the change in emergency patient distribution. Linear regression analysis was used to analyze the effects of COVID-19 infections and Google Trends results on patient numbers. Analysis was performed with Stata 16 and SPSS 25. Graphics were created with Excel, Thinkcell, and Tableau.

\section{Results}

\section{Patient decline}

The average number of patients ( $\bullet$ Fig. 1 ) during workdays fell from 247 before the lockdown to an average of 100 after the start of the first lockdown $(-59.4 \% ; p<0.001)$ and on weekends from 55 to 33 patients $(-40 \% ; p<0.001)$, respectively. For the period before the lockdown, regression analysis showed no significant effect of Google searches or number of COVID-19 infections on the number of patients at the hospital $(p=0.406,95 \% \mathrm{Cl}-1.048$ to 0.431 and $p=0.117,95 \% \mathrm{Cl}-0.076$ to 0.009 ).

During the first 28 days of lockdown, a significant decrease was measured in the number of emergency $(-26.4 \%$; $p<0.001)$, outpatient $(-78.7 \%$; $p<0.001)$, and inpatient $(-53.5 \% ; p<0.001)$ patients as well as of surgeries ( $-61.1 \%$; $<<0.001$; $>$ Fig. 2$)$.

\section{Effect on acute eye diseases}

The number of emergency surgeries decreased from 91 to 81 compared to the elective and urgent surgeries, which decreased from 511 to 171 ( $p<0.001)$. The distribution of emergency patients was significantly different in 2019 and 2020 ( $p=0.024$, - Fig. 3). For patients with a low risk for visual deterioration (risk $=1$ ) and low amount of potential visual deterioration (severity $=1$ ), there was a decline of $-59.2 \%$ compared to $-39.6 \%$ for high-risk/high-severity patients. 


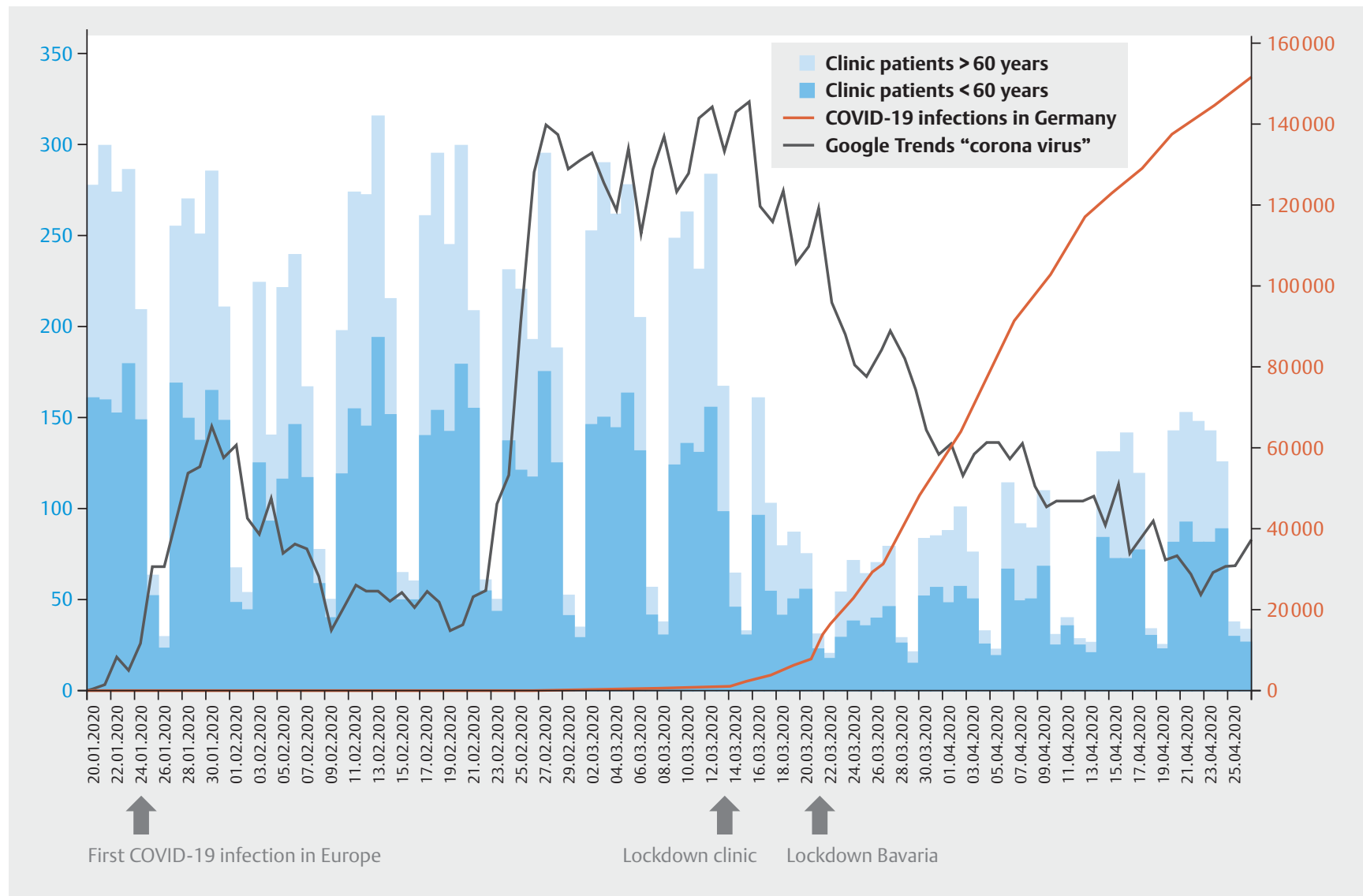

- Fig. 1 The rise in awareness about COVID-19 as shown by the steep increase in Google searches with the term "corona virus" in Germany did not lead to a fall in the number of low-risk (<60 years) or high-risk ( $>60$ years) patients. The lockdown order by the clinic director led to a decline in patient numbers. A week after the internal lockdown, a lockdown by the state of Bavaria was announced. During this period, the number of patients with COVID-19 in Germany was rising continuously.

There was a decrease in patients with an incident, which is a low-, medium-, or high-risk factor, for a future stroke of 27 to 5 , 13 to 8 , and 10 to 9 , respectively $(p=0.003)$.

The number of patients with a change in disability level changed from 14 to 8 for becoming potentially blind, 4 to 1 for having a severely impaired visual acuity, and 10 to 2 for a moderately impaired visual acuity $(p=0.43)$, equaling an overall reduction of $39.3 \%$. Due to this, there was a loss in QALY of 171 QALYs at the university hospital and an estimated loss of 3160 to 24143 QALYs in Germany. The loss in QALY until retirement was $81 \mathrm{QALYs}$, equaling a productivity loss due to visual deterioration of approximately $€ 3.7 \mathrm{M}$ for patients of the eye hospital and an estimation of $€ 69 \mathrm{M}$ to $€ 529 \mathrm{M}$ for eye patients in Germany.

\section{Effect on chronic eye diseases}

The number of visits declined from 304 to 276, 96 to 67, and 257 to 39 for AMD, DR, and glaucoma, respectively. The number of intravitreal injections (used to decrease intraretinal fluid to improve visual acuity) declined from 284 to 231 and 80 to 55 for AMD and $D R$, respectively $(p=0.37)$ and glaucoma surgeries declined from 64 to 27.
The loss in outpatient patients within the analyzed 4 weeks of lockdown compared to 2020 was 3010 patients. The estimated loss in outpatient visits for Germany was, accordingly, 425800 patients. Depending on the ophthalmologist density of each federal state, this would under normal non-pandemic circumstances theoretically lead to a required time period of 7 to 23 weeks to see all patients that have been lost during the total 8 weeks of the analyzed period during the first lockdown ( $\bullet$ Fig. 4).

\section{Optimizing a reschedule scheme after lockdown and guidance for future lockdowns}

The model for single incident diseases would result in a decrease of overall morbidity for the whole population of $56 \%$ if patients are prioritized based on severity only, and a decrease in morbidity of $78 \%$ if prioritized based on severity and urgency ( $\bullet$ Fig. 5 ). The model for chronic diseases would result in a decrease of overall morbidity for high-severity diseases of $31 \%$ if patients are prioritized based on severity only, and a decrease of $33 \%$ if prioritized based on severity and urgency. 

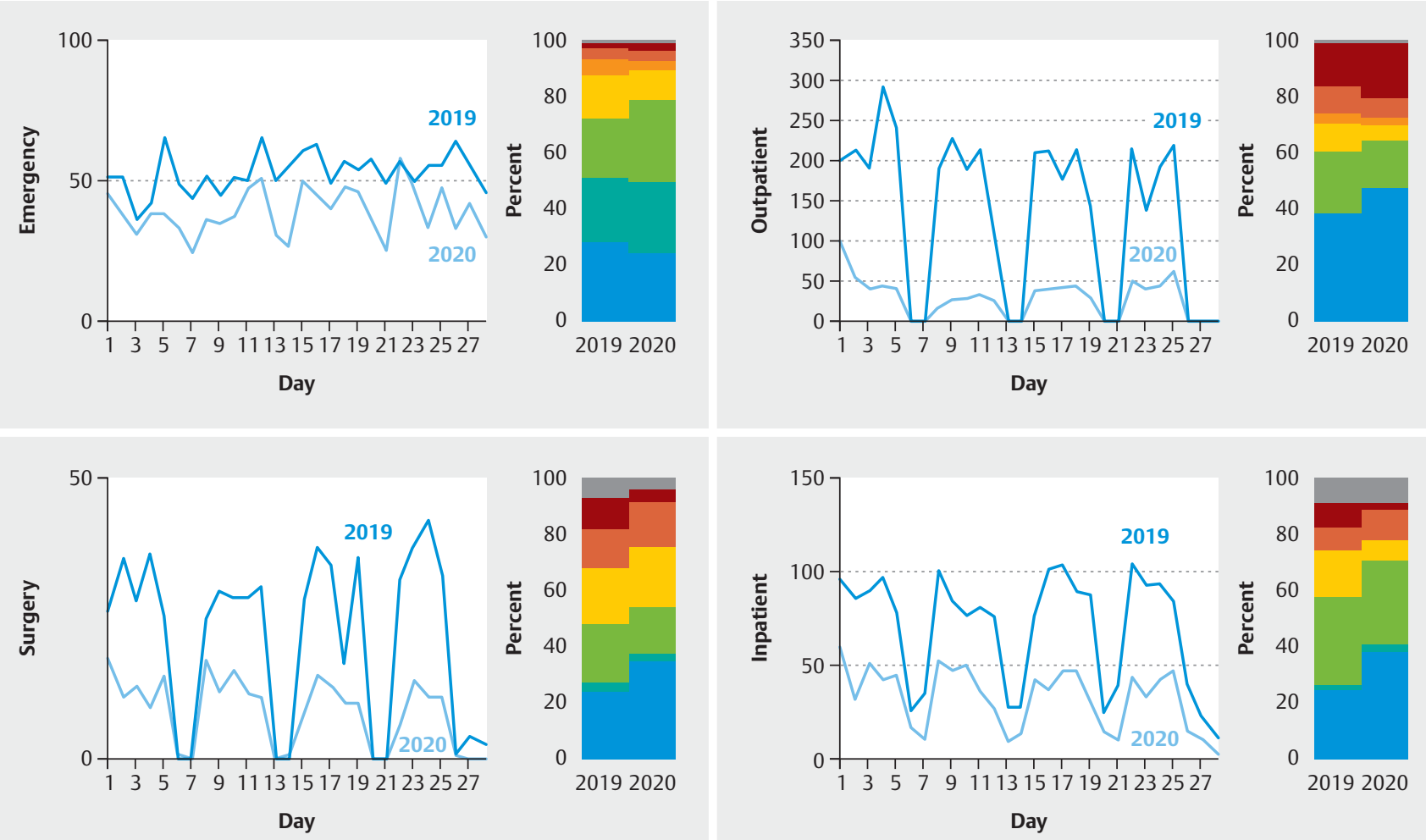

Others Pediatrics and Neuroophthalmology Glaucoma $\square$ Rheumatic diseases and 10 inflammations

Oculoplastic diseases and lid inflammations — Cornea, lens and refraction $\square$ Traumatic diseases and injuries

- Fig. 2 Compared to 2019, a significant decline in the number of emergency, outpatient, and inpatient patients as well as surgeries was measured. In the line graph, the first day is a Monday (further decline during weekends visible, e.g., days 6 and 7 ). The distribution between different subspecialties was similar between 2019 and 2020. For outpatients, there was a relative increase of $22 \%$ for retina and a relative decrease of $-43 \%$ for oculoplastics. For inpatients, a relative decrease of $-63 \%$ could be observed for pediatrics and a relative increase of $47 \%$ for retina. In terms of surgeries, there was a relative decrease of $-73 \%$ for pediatrics, a decrease of $-57 \%$ for oculoplastics, and an increase of $52 \%$ for retina.

\section{Discussion}

Hu et al. [9] observed a correlation between the daily relative searches for COVID-19 via Google and the number of new infections. It could be assumed that increased awareness of the COVID-19 virus would lead to a decrease in the number of patients, as patients want to avoid exposing themselves to an increased risk of infection in hospitals. However, the decrease in the number of patients was unchanged during first 3 weeks of increased awareness of the virus as measured by Google searches. After the hospital's internal lockdown, a dramatic decrease in patients was observed. But not only elective patients whose appointments were cancelled or rescheduled declined, also emergency patients [emergency $(-26.4 \%$; $p<0.001)$, outpatient $(-78.7 \%$; $\mathrm{p}<0.001)$ ]. Although lockdown measures concerning scheduling played the main role, patients' anxieties are reflected in the decline of emergency patients.

Across Germany, a reduction in patient care was observed in eye hospitals during the first months of the pandemic [10,11]. A strength of this study was that all patient files were analyzed by ophthalmologists individually and not automatically by a data system. This way not only a quantitative analysis for the whole population but also a qualitative assessment of each patient's individual situation could be drawn. It is worrying that of emergency cases, not only trivial cases had fallen. The decline in high-severity/high-urgency patients (-39.6\%) was unfortunately also very high compared to the decline in low-severity/low-urgency patients $(-59.2 \%)$. Patients evaluate their situation very subjectively. Ozamiz-Etxebarria [12] reported an increase in anxiety levels of 976 adults in Spain with the onset of the SARS-CoV-2 virus alert. Anxiety can become an important influencing factor. Patients were forced to decide whether it was more important to receive medical care or to protect themselves from a possible COVID-19 infection, a decision which they had to make without all the required medical knowledge. Without knowing all of the required information, this difficult decision was consequently prone to mistakes [13].

Besides providing ophthalmologic treatment, a diagnosis from an ophthalmologist can be helpful in early detection or prevention of internal, sometimes life-threatening diseases such as a stroke. 


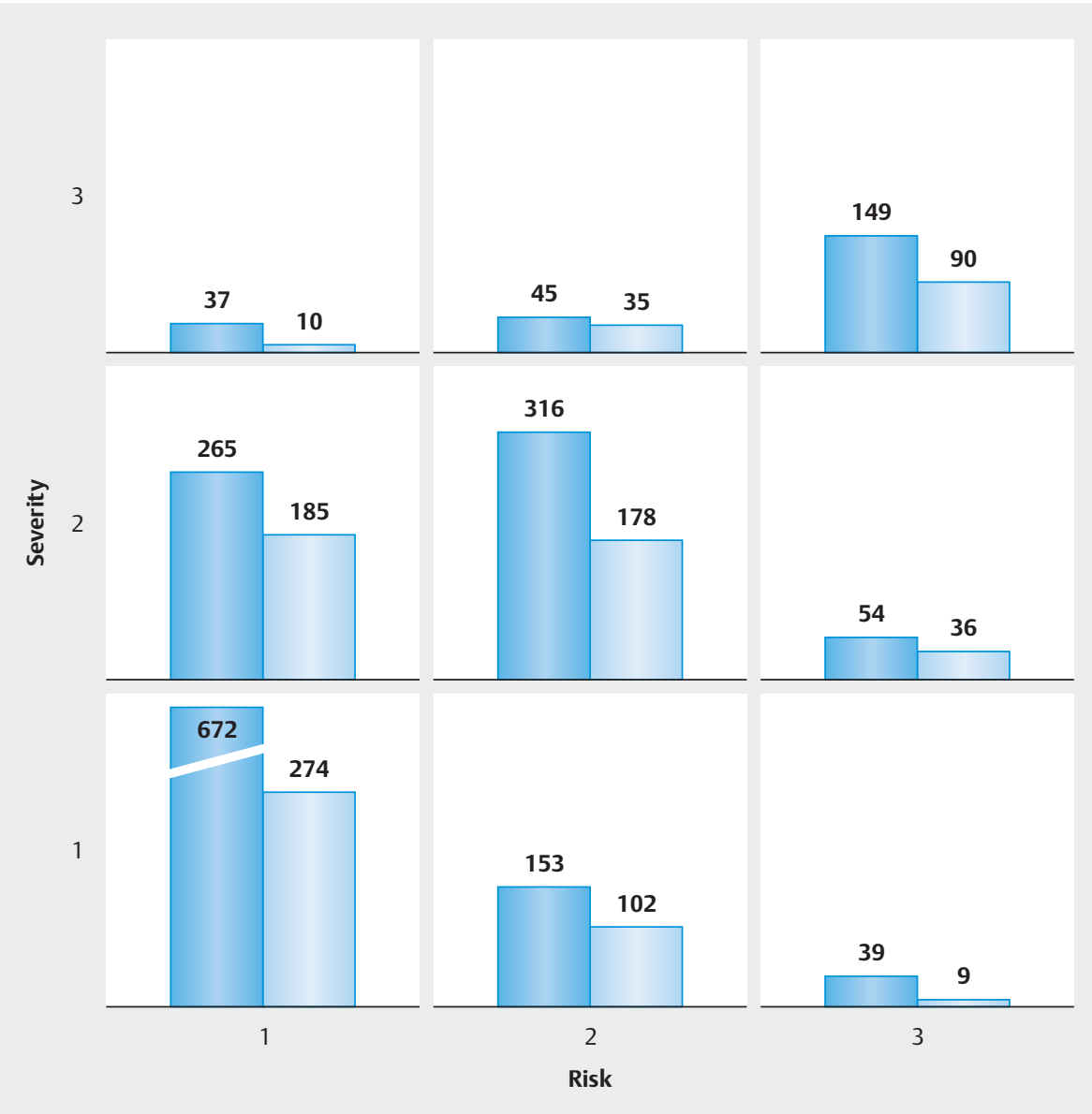

- Fig. 3 Emergency patients were categorized based on their potential loss of visual acuity if not treated within a week (severity, $3=$ high) and the likelihood that this case actually happens (risk, $3=$ high), e.g., a beginning retinal detachment can lead to severe visual deterioration (severity = 3 ) and has a high likelihood that this incidence will occur if left untreated for a week (risk =3).

In CP, there were significantly less patients with hints for a potential cardiovascular issue as during NCP. For example, non-controlled arterial hypertension can become noticeable for the first time by conjunctival bleeding (hyposphagm) [14] or Amaurosis fugax, a TIA of the central retinal artery, which has a 3 to $20 \%$ risk for stroke and central retinal artery occlusion and a $44 \%$ risk for a second ischemic event within the next week [15]. Early recognition and treatment of the underlying diseases is crucial and can prevent severe courses. Similar to our findings, different neurology departments reported on a dramatic decline in strokes during the first lockdown [16-19]. Not only did they observe a reduction in the total number of events of $23-45 \%$, but also a reduction in referrals [17] and an increase in severe cases $[16,18,19]$. Naccarato et al. even reported a worse functional outcome after reperfusion compared to an NCP [18]. The decline is thought to be due to patients' fear of contracting COVID-19 in a hospital setting, which led them to report symptoms late.

Impaired vision can lead to loss in QALY, loss in productivity, and the need for care, with a dramatic increase in costs for society $[7,20]$. Our projected loss of 3160 to 24143 QALYs nationwide is a relatively high number considering that only 4 weeks of lockdown were evaluated. Furthermore, our results show the situation due to undertreatment in eye care. Many diseases have a much higher utility value than blindness (e.g., stroke 0.5 to 0.7 ) and can lead to a much stronger change in QALY. Similarly, in other specialties, a high number of patients, who had a mild preceding incident for which they did not get medical care during the lockdown, might now suffer severely from a potential secondary incident that could have been prevented.

Unprepared for a pandemic, appointments were cancelled without sufficiently outweighing the loss in medical care against the goal to reduce viral spread. This led to a marked decrease in treatments of chronic diseases like AMD or glaucoma. Lim et al. [21] reported a considerably worse outcome for patients with AMD who receive delayed therapy. It will probably take 7 to 23 weeks under normal circumstances, depending on the region, to catch up on missed appointments. Foot and MacEwen showed that $72 \%$ of patients with a median delay in consultation of 22 weeks for AMD, DR, and glaucoma experienced a permanent reduction in visual acuity [22]. The postponement of medical appointments caused by the COVID-19 pandemic gives reason to expect similar losses.

Furthermore, the proportion of physicians working in private clinics has risen steadily in recent years [3]. At our university clinic, 


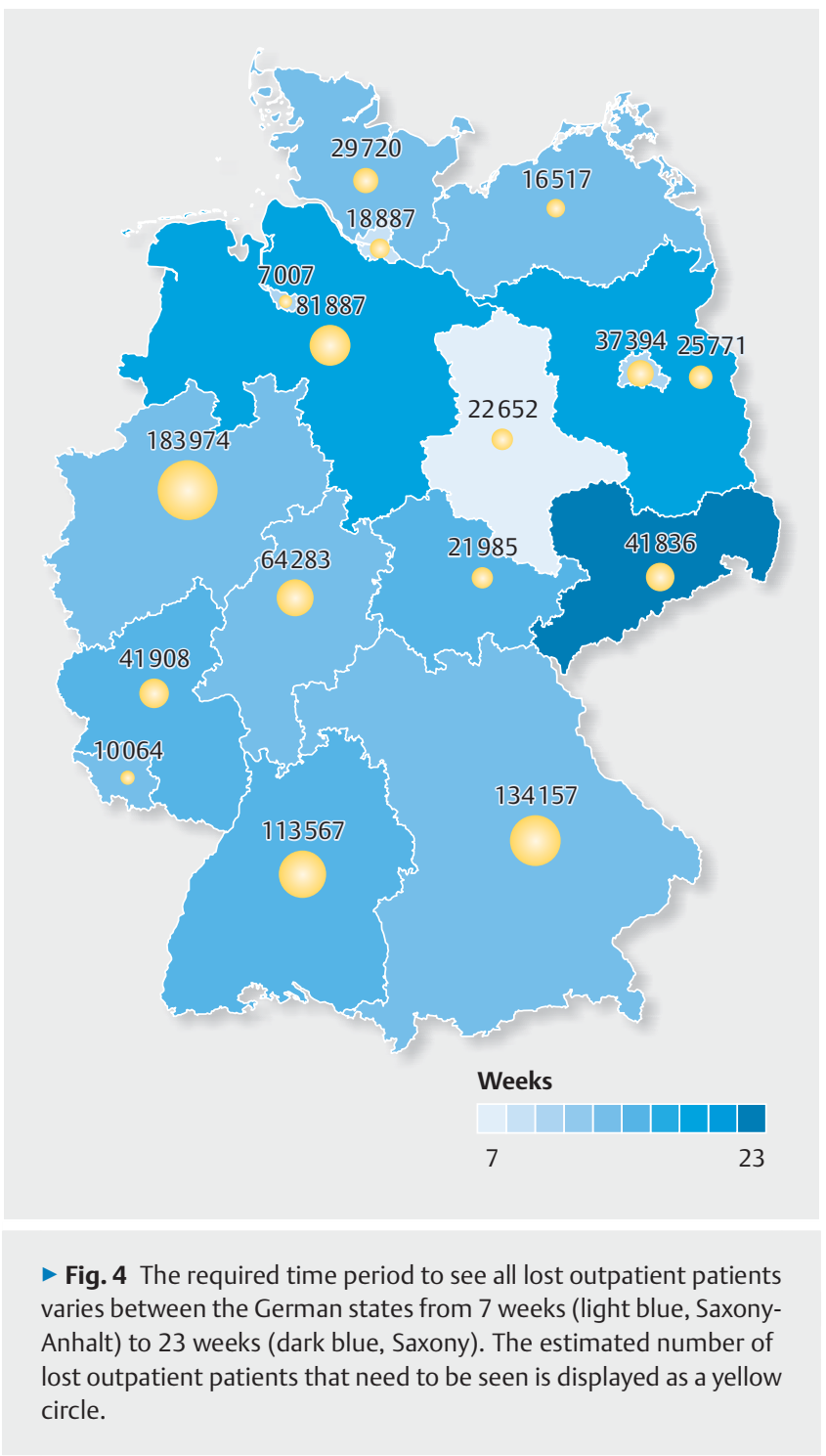

the number of low and medium urgent elective surgeries declined by $67 \%$. A similar decline in elective cases in the private sector, if not even higher, would cause a dramatic drop in revenues. It is essential for the private clinics to remain profitable in order to maintain medical care. This was underlined already in the beginning of the crisis by Cavallo and Forman [23] in their report on the dramatic changes for radiology practices. State financial support was considered, but they also appeal to the heads of private institutions to consider restructuring. The ongoing pandemic led to higher costs due to the hygienic concepts and lower revenues due to lower utilization of the normal services. According to a current report, several clinics and practices in Germany fear going insolvent without governmental aid, which would lead to further deterioration in medical care [24].

Even though the number of infections was declining with the first lockdown, experts [25] already in the beginning of the pandemic believed that new waves of infection were ahead of us. Various measures [26-28] were proposed to minimize the spread of infection and possible direct health damage in that scenario. But unfortunately, it was not possible to protect the population from a second wave. In autumn, the crisis was back and led to a second lockdown in Germany, which started in December. Due to a lack of intensive care capacity, many hospitals all over Germany had difficulties maintaining regular care during the second pandemic wave because they had to provide additional staff for the Covid19 patients $[29,30]$. Fortunately, at the University Eye Hospital Munich, a drastic cancellation of appointments as during the first lockdown was not necessary. The schedule was adjusted to ensure spacing in the waiting areas; due to the shortage of anesthesia staff, elective general anesthesia surgeries had to be postponed sometimes. Even though there were now suggestions how to proceed in a pandemic, especially regarding hygiene measures [10], there still remained uncertainty as to the necessary restrictions in patient care. The uncertainty regarding the correct behavior during lockdown is described by numerous physicians [31] - which patients have to be seen, how much do I have to protect the patients and also the staff?

Already at the beginning of the first lockdown, voices rose to remind us that the treatment of other critical diseases during the pandemic cannot be forgotten and made first suggestions on the prioritization of patients [1]. In contrast, Sæther et al. [32] recommend a "first come, first serve" approach to reduce long waiting times. Over the last months, diverse suggestions from different medical disciplines came up $[33,34]$. Our developed model for patient selection according to prioritization through urgency and severity showed, that above all, consideration of severity for both single incident and chronic diseases plays a decisive role. Our model has not yet been evaluated in a clinical setting, but it is reasonable to believe that the mathematical benefit of prioritization as opposed to randomization will also be evident in real life once put in place.

\section{Limitations}

To estimate the undersupply of patients in CP the difference of treated patients of 2019 to 2020 was calculated. This is an estimation of potentially lost patients, not the actual loss. It is possible that the counted difference between $\mathrm{CP}$ and NCP period results from the fact that patients consulted smaller private practices in order to avoid being infected in a big hospital. However, we believe due to the complete closure of many private practices during lockdown, critical care should have even increased in our hospital in relation to other places. Nevertheless, it must be taken into account that the loss of visits per ophthalmologist due to the first lockdown may vary between different ophthalmic facilities and regions. Therefore, the extrapolation for Germany based on our hospital's data might be falsified by regional and facility differences. Multicenter evaluations will be necessary to confirm our results. In a large German-wide survey, Hattenbach et al. found a limitation in ophthalmic treatment during the first period of the first lockdown in both conservative and surgical settings. Most of the participating ophthalmologists reported restricted treatment (63.5\%), some only offered emergency treatment (27.7\%) [11].

A possible visual impairment due to non-treatment was estimated as well, because an actual omitting of therapy to monitor its effectiveness is inconceivable. To achieve a relatively realistic 


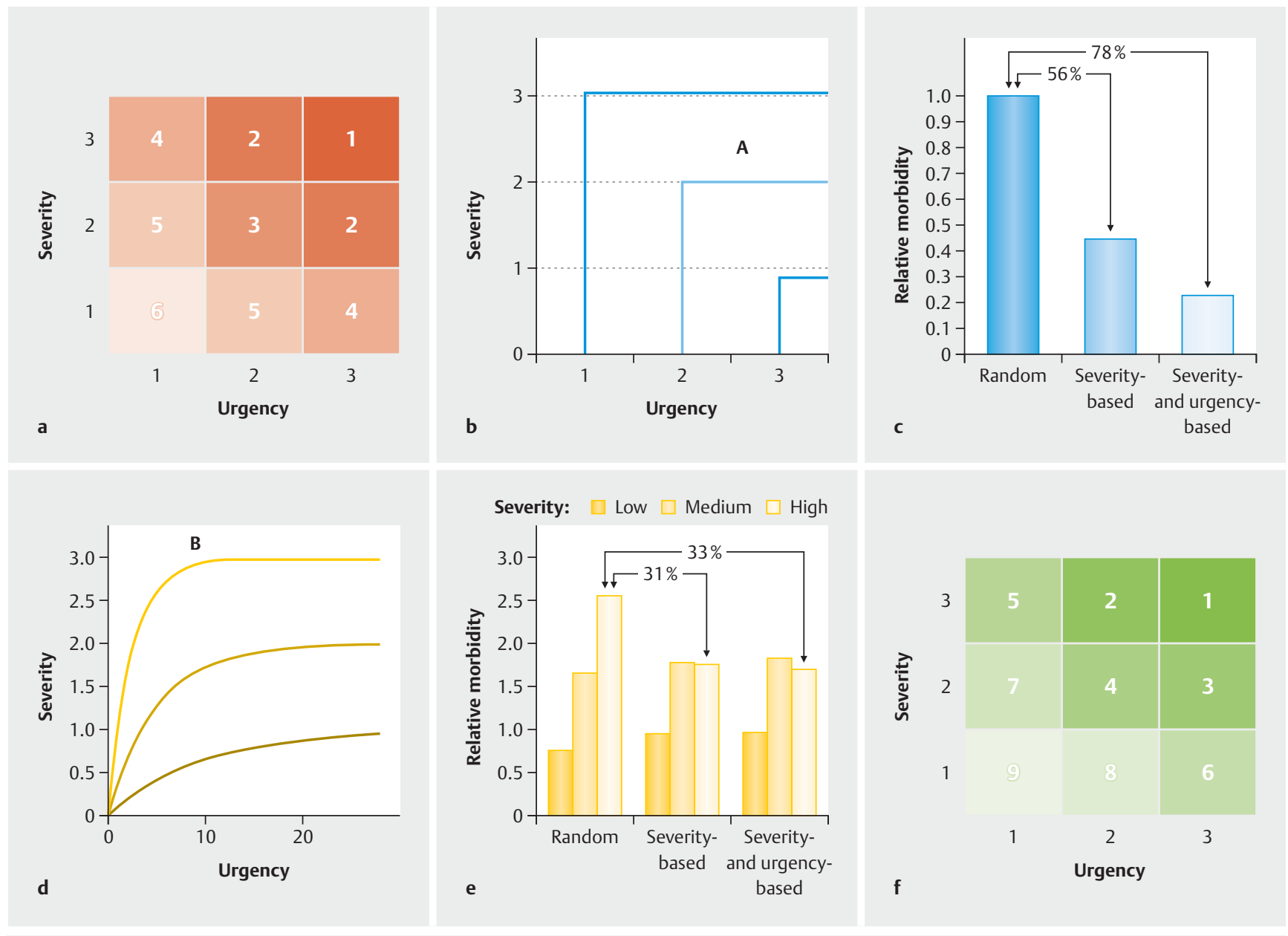

- Fig. 5 Patients could be prioritized based on the product of urgency and severity of a disease to decrease overall population morbidity (red graphic, a). This would mean that patients with high severity $(=3)$ and high urgency $(=3)$ would be seen first, as indicated by the " 1 " in the upper right box and accordingly, patients with low severity $(=1)$ and low urgency $(=1)$ would be seen last, as indicated by the " 6 " in the lower left box. Patients with high severity $(=3)$ and low urgency $(=1)$ achieve the same prioritizing level as patients with low severity $(=1)$ and high urgency $(=3)$, indicated by " 4 " in the upper left and lower right boxes. To further analyze a single incident (like, e.g., central retinal artery occlusion, blue graphs, b, c) and chronic diseases (like, e.g., diabetic retinopathy, yellow graphs, d, e), a simulation model was calculated. Patients with different severity and urgency levels were analyzed. For example, patient A (b) has a medium severity (2) and medium urgency (2) single incident disease and patient B (c) has a high severity (3) and high urgency (3) chronic disease (as seen by a plateau curve). The model showed that prioritization based on severity results in a high level of decrease in population morbidity, especially in single incident diseases ( $-56 \%$ by severity prioritization and $-78 \%$ by severity and urgency prioritization, $c$ ) and high severity chronic diseases $(-31 \%, e)$. Therefore, patients should be prioritized based on the product of severity and urgency with higher consideration of severity in case the product of severity and urgency is equal for two patients (green graphic, $\mathrm{f}$ ). This means after having seen patients with high severity, high urgency (upper right green box, "1"), patients with high severity and medium urgency should be seen ("2").

estimation, risk in every patient's situation was evaluated from two independent senior ophthalmologists.

Our results are not representative for individual regions of Germany, but rather for similar metropolitan areas. This has to be considered in the projection for Germany.

This is a single-center study and, consequently, it might be difficult to draw conclusions on a national level. However, the number of patients included in this study is quite high for such a short period of time due to the large size of the clinic and allows to draw assumptions on a larger scale. Proof that the numbers presented might be a good reflection of the national situation is the decline in overall cases, as reported by the largest health insurance company in Germany (26.3 million members), e.g., - 66\% for muscu- loskeletal diseases, $-51 \%$ for respiratory diseases, and $-41 \%$ for cardiovascular diseases (no report on ophthalmologic cases) [35]. We found a decline of - 59\% for overall ophthalmology patients. Concerning is the reported decline of $-30 \%$ in patients with a stroke, which is in line with our loss in patients with a medium- to high-level hint for a stroke of $-26 \%$.

\section{Supplementary material}

Supplement 1: Calculation of weeks required to see missed patients. Supplement 2: Development of a model to prioritize patient visits during and after lockdown. 


\section{Acknowledgements}

We thank Alison Jefferson for proofreading the manuscript for any language errors.

\section{Conflict of Interest}

The authors declare that they have no conflict of interest.

\section{References}

[1] Baker T, Schell CO, Petersen DB et al. Essential care of critical illness must not be forgotten in the COVID-19 pandemic. Lancet 2020; 395: 12531254. doi:10.1016/S0140-6736(20)30793-5

[2] Pfefferbaum B, North CS. Mental health and the Covid-19 pandemic. N Engl J Med 2020; 383: 510-512. doi:10.1056/NEJMp2008017

[3] Bundesärztekammer. Ärztestatistik zum 31. Dezember 2018 (In German). Accessed May 20, 2020 at: https://www.bundesaerztekammer. de/fileadmin/user_upload/downloads/pdf-Ordner/Statistik2018/ Stat18AbbTab.pdf

[4] European Centre for Disease Prevention and Control. Latest information on COVID-19. (In English). Accessed May 12, 2020 at: https://www.ecdc. europa.eu/en

[5] Google Trends. Erkunden/Corona Virus. In German. Accessed May 11, 2020 at: http://trends.google.de/trends

[6] World Health Organization. Blindness and vision impairment. (In English). Accessed May 12, 2020 at: https://www.who.int/news-room/factsheets/detail/blindness-and-visual-impairment

[7] Wittenborn JS, Zhang X, Feagan CW et al. The economic burden of vision loss and eye disorders among the United States population younger than 40 years. Ophthalmology 2013; 120: 1728-1735. doi:10.1016/j. ophtha.2013.01.068

[8] Sharma S, Brown GC, Brown MM et al. Converting visual acuity to utilities. Can J Ophthalmol 2000; 35: 267-272. doi:10.1016/s0008-4182 (00)80077-0

[9] Hu D, Lou X, Xu Z et al. More effective strategies are required to strengthen public awareness of COVID-19: Evidence from Google Trends. J Glob Health 2020; 10: 011003. doi:10.7189/jogh.10.011003

[10] Hattenbach LO, Reinhard T, Walter P et al. [Crisis management strategies of hospitals during the pandemic]. Ophthalmologe 2020; 117 : 652-658. doi:10.1007/s00347-020-01162-x

[11] Hattenbach LO, Heinz P, Feltgen $\mathrm{N}$ et al. [Impacts of the SARS-CoV-2 pandemic on ophthalmic care in Germany]. Ophthalmologe 2020; 117 : 892-904. doi:10.1007/s00347-020-01220-4

[12] Ozamiz-Etxebarria N, Dosil-Santamaria M, Picaza-Gorrochategui M et al. Stress, anxiety, and depression levels in the initial stage of the COVID-19 outbreak in a population sample in the northern Spain. Cad Saude Publica 2020; 36: e00054020. doi:10.1590/0102-311X00054020

[13] Kittleson MM. The Invisible Hand - Medical Care during the Pandemic. N Engl J Med 2020; 382: 1586-1587. doi:10.1056/NEJMp2006607

[14] Doshi R, Noohani T. Subconjunctival Hemorrhage. 2020 Nov 17. In: StatPearls [Internet]. Treasure Island (FL): StatPearls Publishing; 2021. Accessed July 5, 2021 at: https://pubmed.ncbi.nlm.nih.gov/31869130/

[15] Park SJ, Choi NK, Yang BR et al. Risk and Risk Periods for Stroke and Acute Myocardial Infarction in Patients with Central Retinal Artery Occlusion. Ophthalmology 2015; 122: 2336-2343.e2 doi:10.1016/j.ophtha. 2015.07.018

[16] Wu Y, Chen F, Wang Z et al. Reductions in hospital admissions and delays in acute stroke care during the pandemic of COVID-19. Front Neurol 2020; 11: 584734. doi:10.3389/fneur.2020.584734
[17] Bres Bullrich M, Fridman S, Mandzia JL et al. COVID-19: Stroke Admissions, Emergency Department Visits, and Prevention Clinic Referrals. Can J Neurol Sci 2020; 47: 693-696. doi:10.1017/cjn.2020.101

[18] Naccarato M, Scali I, Olivo S et al. Has COVID-19 played an unexpected "stroke" on the chain of survival? J Neurol Sci 2020; 414: 116889. doi:10.1016/j.jns.2020.116889

[19] Kristoffersen ES, Jahr SH, Thommessen B et al. Effect of COVID-19 pandemic on stroke admission rates in a Norwegian population. Acta Neurol Scand 2020; 142: 632-636. doi:10.1111/ane.13307

[20] Chuvarayan Y, Finger RP, Koberlein-Neu J. Economic burden of blindness and visual impairment in Germany from a societal perspective: a cost-ofillness study. Eur J Health Econ 2020; 21: 115-127. doi:10.1007/s10198019-01115-5

[21] Lim JH, Wickremasinghe SS, Xie J et al. Delay to treatment and visual outcomes in patients treated with anti-vascular endothelial growth factor for age-related macular degeneration. Am J Ophthalmol 2012; 153: 678-686, 686.e1-2. doi:10.1016/j.ajo.2011.09.013

[22] Foot B, MacEwen C. Surveillance of sight loss due to delay in ophthalmic treatment or review: frequency, cause and outcome. Eye (Lond) 2017; 31: 771-775. doi:10.1038/eye.2017.1

[23] Cavallo J], Forman HP. The economic impact of the COVID-19 pandemic on radiology practices. Radiology 2020: 296: e141-e144. doi:10.1148/ radiol.2020201495

[24] Telgheder M. Kliniken senden Notruf: Ohne neue Hilfe drohen ab dem Frühjahr Insolvenzen. (In German). Accessed April 20, 2021 at: https:// www.handelsblatt.com/technik/medizin/gesundheitswesen-klinikensenden-notruf-ohne-neue-hilfen-drohen-ab-dem-fruehjahrinsolvenzen/26847658.html?ticket=ST-6143299-mdsp9BZy]dYYF ZCd2HPl-ap2

[25] Xu S, Li Y. Beware of the second wave of COVID-19. Lancet 2020; 395: 1321-1322. doi:10.1016/S0140-6736(20)30845-X

[26] Heymann DL, Shindo N. COVID-19: what is next for public health? Lancet 2020; 395: 542-545. doi:10.1016/S0140-6736(20)30374-3

[27] Gates B. Responding to Covid-19 - A Once-in-a-Century Pandemic? N Engl J Med 2020; 382: 1677-1679. doi:10.1056/NEJMp2003762

[28] Inglesby TV. Public Health Measures and the Reproduction Number of SARS-CoV-2. JAMA 2020; 323: 2186-2187. doi:10.1001/jama.2020. 7878

[29] [Anonymous]. Mehr Covid-19-Patienten auf Intensivstationen. (In German). Accessed April 20, 2021 at: https://www.aerzteblatt.de/ nachrichten/119092/Mehr-COVID-19-Patienten-auf-Intensivstation

[30] Baum G, Gaß G. Krankenhäuser im zweiten Pandemiejahr/Deutsche Krankenhausgesellschaft. [In German]. Accessed April 20, 2021 at: https://www.dkgev.de/fileadmin/default/Mediapool/1_DKG/1.7_ Presse/1.7.1_Pressemitteilungen/2021/2021-01-26__Folien_PK.pdf

[31] Nair AG, Gandhi RA, Natarajan S. Effect of COVID-19 related lockdown on ophthalmic practice and patient care in India: Results of a survey. Indian J Ophthalmol 2020; 68: 725-730. doi:10.4103/ijo.IJO_797_20

[32] Saether SMM, Heggestad T, Heimdal JH et al. Long Waiting Times for Elective Hospital Care - Breaking the Vicious Circle by Abandoning Prioritisation. Int J Health Policy Manag 2020; 9: 96-107. doi:10.15171/ ijhpm.2019.84

[33] Deora H, Sadashiva N, Tripathi M et al. The Aftermath of COVID-19 Lockdown - Why and How Should We Be Ready? Neurol India 2020; 68: 774791. doi:10.4103/0028-3886.293471

[34] Curigliano G, Cardoso MJ, Poortmans P et al. Recommendations for triage, prioritization and treatment of breast cancer patients during the COVID-19 pandemic. Breast 2020; 52: 8-16. doi:10.1016/j.breast. 2020.04.006

[35] Erste Ergebnisse aus den Auswertungen zu Covid-19- Patienten. [In German]. Accessed May 12, 2020 at: https://www.aok-bv.de/imperia/md/ aokbv/hintergrund/dossier/krankenhaus/aok-fallzahlen_202019.pdf 